\title{
Non-Kolmogorov features of differential image motion restored from the Multichannel Astrometric Photometer data
}

\author{
P. F. Lazorenko` \\ Main Astronomical Observatory, National Academy of Sciencies of Ukraine, Zabolotnogo 27, 03680 Kyiv-127, Ukraine
}

Received 14 March 2002 / Accepted 10 September 2002

\begin{abstract}
In this paper we present a new restoration of atmospheric turbulent parameters using published data on image motion observations with the Multichannel Astrometric Photometer. These data have been previously analyzed by Han (1989) whose estimate of the power of the phase structure function $\alpha=1.64$ was close to the $5 / 3$ value expected for the Kolmogorov atmospheric turbulence. Investigating the temporal image motion spectrum, we show, however, that the experimental data do not follow predictions of the Kolmogorov model. The best fitting of the observed spectrum is achieved with $\alpha=1.9-2.0$, which is typical of non-classic phase distortions. The outer scale of turbulence was found to be $1800 \mathrm{~m}$ or longer. Expressions for the non-Kolmogorov temporal spectrum of differential image motion allowing for a finite outer scale length are given.
\end{abstract}

Key words. atmospheric effects - turbulence - methods: data analysis - astrometry

\section{Introduction}

Atmospheric turbulence is a factor that strongly deteriorates image quality observed through the atmosphere, and causes image motion, which affects the position of stars. Atmospheric effects have been intensely studied for some decades. The basic assumption used in most theoretical considerations (e.g. Fried 1965; Tatarsky 1961) was that atmospheric turbulence follows the Kolmogorov theory of a fully developed 3D turbulence. Predictions made on this basis, in particular for image motion, usually give correct results and fit experimental data well (Lindegren 1980; Martin 1987; Sarazin \& Roddier 1990).

Some studies, however, testify that turbulence sometimes may display significant deviations from its classical behavior. Thus, meteorological studies of the temperature and wind spatial spectra measured in the turbulent atmospheric layers often indicate strong deviations from the classic behaviour (Vinnichenko et al. 1976; Hogstrom et al. 1998). A short bibliography of meteorological, theoretical and astronomical data testifying that the non-Kolmogorov turbulence actually occurs was given by Lazorenko (2002, hereinafter Paper I). Concerning distortions of the turbulent light phase, these data suggest that both the power $\alpha=1+p$ of a phase structure function and a slope of $-3-p$ in Eq. (3) for the spatial power spectral density (PSD) of phase fluctuations should be considered as the quantities that vary from a sample to sample. The value of parameter $p$ which is equal to $2 / 3$ in case of the Kolmogorov turbulence depends on physical conditions in the given

\footnotetext{
* e-mail: laz@mao.kiev.ua
}

atmospheric layer, and may vary in a wide range from $-1 / 3$ to 1 . Moreover, it is a function of spatial scale. Thus, while on scales of $1-300 \mathrm{~m}, p$ varies normally from $1 / 3$ to $2 / 3$, with a mean value of about 0.5 , at longer $200-1000 \mathrm{~m}$ scales the PSD often shows saturation described by von Karman statistics. Here $p$ decreases, sometimes to negative values.

In analytical expressions derived for the variance $\Delta^{2}$ of differential image motion, Lindegren (1980) used a variable term $\alpha=1+p$ representing the power of a phase structure function. His numerical estimates originally were given in dual form: with $\alpha=5 / 3$ for the Kolmogorov type of turbulence and $\alpha=3 / 2$ consistent with the model of the absolute image motion spectrum suggested by Hog (1967). Lindegren did not specify which of the above estimates is to be used. In many subsequent studies, however, the variance of differential image motion was calculated only with $\alpha=5 / 3$, or $p=2 / 3$. Besides being theoretically substantiated, the correctness of the $p=2 / 3$ value experimentally was confirmed later by Han (1989), whose analysis of observational data supported Kolmogorov distortions of a turbulent wave-front. In his study, Han derived differential image motion characteristics from the Multichannel Astrometric Photometer (MAP) observational data, and obtained an estimate $p=0.64$, very close to the Kolmogorov 2/3 value. Hence this result was often referred to as a direct experimental proof of the applicability of a classic theory of turbulence to astronomical measurements.

In this paper we present a new interpretation of the MAP image motion statistics that is described in Sect. 2. Our analysis is based on rigorous expressions for the differential image 
motion spectrum derived in Sect. 3 and is valid for a variable value of $p$. In Sect. 4 we use these expressions for a simulation of the MAP data, and restore parameters of turbulence. Here, we show that the experimental data do not follow predictions of the conventional theory, and establish the best estimate $0.9-1.0$ for $p$. In Sects. 5 and 6 we verify the reliability of the model by applying it to simulation of the observed dependences of the variance $\Delta^{2}$ on the star separation and exposure. In Sect. 7 we consider effects which should be present in the temporal power spectrum of differential image motion in the case of a finite outer scale of turbulence, and establish its feasible values.

\section{Description of the data discussed}

In this investigation, we refer to observational data given by Han (1989) and presented largely in the form of graphs. Below we give some necessary information important for an understanding of our analysis.

Observations were carried out with the Multichannel Astrometric Photometer (MAP). The telescope (Gatewood $1987)$ is a refractor $(D=0.78 \mathrm{~m})$ used for determination of parallaxes and proper motions. The principal metric element of the MAP is a Ronchi ruling consisted of a set of transparent lines and opaque spaces of equal width. The ruling, moving across a focal plane, produces modulation of light from stars. The scanning of star images is performed in two directions. First, the ruling moves along the $x$-axis (right ascention), then images are scanned perpendicularly, along the $y$-axis (declinations). The total duration of each scan is about $10 \mathrm{~min}$.

On a temporal scale, one period of the ruling corresponds to the $\Delta t=1.6 \mathrm{~s}$ interval, and some effective time of image exposure $T_{1}$ which depends on the image size. Thus, in the event of a dot element image, $T_{1}=\Delta t / 2$, increasing to $T_{1} \simeq \Delta t$ for a blurred image. With image sizes normally equal to a half of the ruling period, $T_{1}$ is in the range between these two extremes. In further calculations we assumed that approximately $T_{1}=\frac{3}{4} \Delta t=1.2 \mathrm{~s}$. The discreteness of measured data allows averaging of star positions over some $m$ sequential periods. In that way the effective exposure is incresed to $T_{1}+(m-1) \Delta t$ or

$$
T=m \Delta t-\frac{1}{4} \Delta t
$$

In the original paper, the variance of differential image motion $\Delta^{2}$ was found as the average of $\Delta_{x}^{2}$ and $\Delta_{y}^{2}$ variances representing image motion along the $x$ and $y$ axes. The observed dependence of measured $\Delta$ values versus a separation $\rho$ between the two stars is reproduced in Fig. 1 for the integration time $T=10 \mathrm{~s}$. In log-log coordinates the measured points are fitted by a straight line 1 with a slope of 0.32 obtained by Han and is near the expected $p / 2=1 / 3$ value for the Kolmogorov turbulence (Lindegren 1980). One may notice, however, that due to insufficient statistics and, especially, the narrow range of $\rho$ variation, the fitting is not quite reliable: the measured points can be approximated equally well by lines with different slopes. Moreover, a linear (in logarithmic plot) approximation of the function $\Delta(\rho) \simeq \rho^{p / 2}$ is valid only at the asymptotic condition $\rho h \ll V T$ (here $h$ and $V$ stand for the height and wind velocity of the turbulent layer) which is not evident

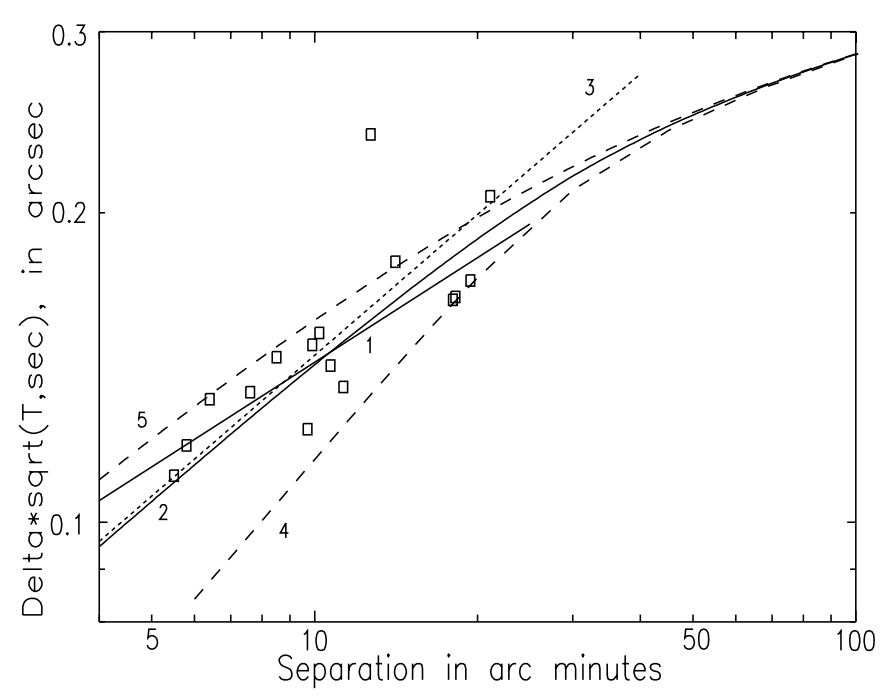

Fig. 1. Dependence of the variance $\Delta$ on star separation $\rho$ at $T=10 \mathrm{~s}$. Observed data (Han 1989) (squares) with a linear approximation (1); curvilinear function (2) calculated with $p=0.94$, and the asymptotic power law (3) expected at long $T \gg \rho h / V$; lower (4) and upper (5) boundaries for measured points at positional angles of star pairs $\psi=10^{\circ}$ and $\psi=90^{\circ}$ correspondingly.

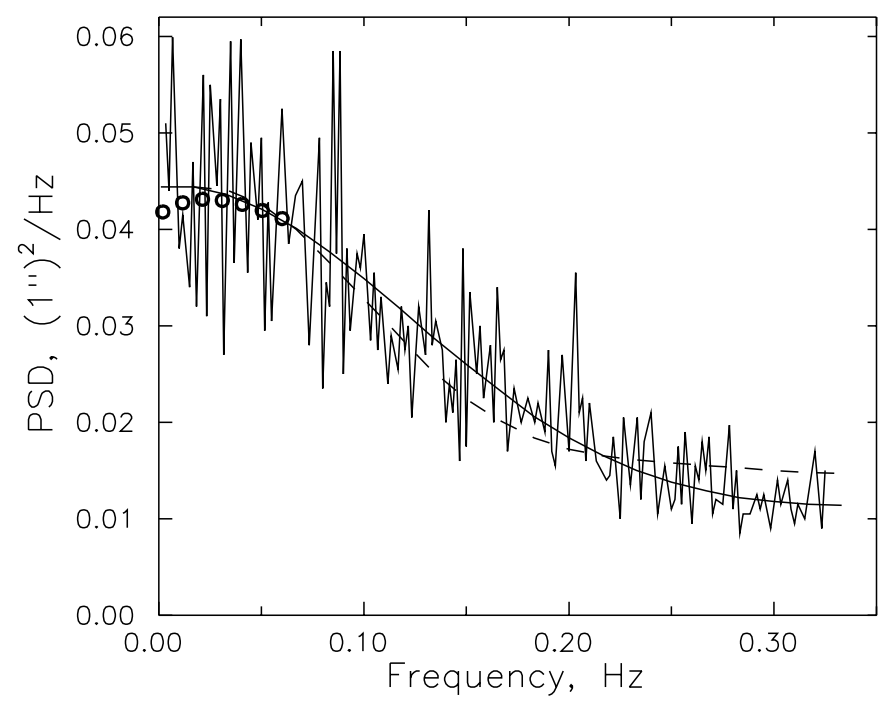

Fig. 2. Temporal image motion PSD versus frequency $f$ at $T=1.2 \mathrm{~s}$ and $\rho=10^{\prime}$. Observed spectrum $G(f)$ (broken line); best simulated $\hat{G}(f)$ with $p=0.94$ (solid), $p=2 / 3$ (dashed) and with allowance for an outer scale $L_{0}=1800 \mathrm{~m}$, see Sect. 7 (open circles).

and, as we testify below, is incorrect. In the non-asymptotic case, the function $\Delta(\rho)$ is expanded into a power series of $\rho$ as $\Delta^{2}=a_{1} h^{p} \rho^{p}(V T)^{-1}-a_{2} h^{2} \rho^{2}(V T)^{-3+p}+\ldots$ where $a_{1}, a_{2}$ are some constants dependent on $p$ and the intensity of turbulence (Eq. (24) of Paper I). Because the second $\rho^{2}$ term of the expansion is negative, the slope of the function $\Delta(\rho)$ decreases with $\rho$. For that reason, the data in Fig. 1 only set a restriction $p>0.64$.

The key information is contained in the measured temporal PSD of differential image motion $G(f)$ which is reproduced in Fig. 2 (broken line) with a high spectral resolution up to 
the Nyquist frequency $f_{\mathrm{N}}=1 /(2 \Delta t) \approx 0.32 \mathrm{~Hz}$. The PSD was derived with the shortest sample rate $\Delta t=1.6 \mathrm{~s}$ and effective integration time $T=1.2 \mathrm{~s}$; these data are an average over all observations and correspond to the mean stellar separation $10^{\prime}$. Owing to a rather complicated shape, this plot is very informative, and when used in the fitting procedure allows one to recover original parameters of the turbulent wave-front distortions.

\section{A model of the temporal power spectrum of differential image motion}

To find the model spectrum $g(f)$ related to the measured function $G(f)$, we start with a common expression for the 2D spatial power spectrum $F(u, v)$ of differential image motion derived in Paper I. This expression has a form

$$
F(u, v)=F_{\phi}(q) Q(u, v)
$$

of the product of two functions: first is the 2D spatial PSD of a turbulent phase $F_{\phi}(q)$ entering the telescope pupil and which is generated by some atmospheric layer at a height $h$, and the second is a filter function $Q(u, v)$ that accounts for observational (non-atmospheric) details alone: exposure, telescope aperture, the structure and size of stellar configurations measured etc. Here $u, v$ are spatial frequences, and $q=\sqrt{u^{2}+v^{2}}$ is a circular frequency measured in units of $\mathrm{m}^{-1}$. The function

$$
F_{\phi}(q)=c_{\phi} q^{-3-p}
$$

describes 2D fluctuations of phase, $c_{\phi}$ is a normalizig factor, and $p$ is the parameter to be established in this study. A model with an outer scale will be discussed later in Sect. 7.

Expression (2) describes filtration of atmospherically induced wave front distortions, which accur in the process of observations, and is valid, generally, for any phase-related quantity. In our case, a measured quantity is the angular separation $\rho$ between the two stars, and its fluctuation $\Delta$. As shown in Paper I, in this case the function $Q(u, v)$ is expressed as a product of four elemental filter functions $Q_{1} \ldots Q_{4}$ originating from the following effects and procedures that are peculiar to differential astrometric techniques:

1) Displacement of star images caused by the gradient of the wave-front in the direction of measurements that forms some angle $\theta$ with the $u$-axis. If the latter is directed along the wind vector, then $Q_{1}(u, v)=\lambda^{2}(u \cos \theta+v \sin \theta)^{2}$;

2) Averaging due to a finite exposure: $Q_{2}(u, v)=$ $\operatorname{sinc}^{2}(\pi V T u)$

3) Averaging over the telescope aperture: $Q_{3}(u, v)=$ $\operatorname{somb}^{2}(\pi D q)$;

4) Formation of a difference in the two points of a phase screen at which it is crossed by light beams passing from the stars. The relative position of the points is defined by the star separation $h \rho$ and the positional angle $\psi$ measured with reference to the wind. Then $Q_{4}(u, v)=2\{1-\cos [2 \pi \rho h(u \cos \psi+$ $v \sin \psi)]\}$. In the above expressions $\lambda$ is wavelength, $\operatorname{sinc}(z)=$ $\sin (z) / z, \operatorname{somb}(z)=2 J_{1}(z) / z$, and $J_{1}$ is the Bessel function of the order 1 .
With these expressions, Eq. (2) is rewritten as

$$
\begin{aligned}
F(u, v)= & \lambda^{2} c_{\phi} q^{2} \operatorname{sinc}^{2}(\pi V T u) \operatorname{somb}^{2}(\pi D q) \\
& \times\{1-\cos [2 \pi \rho h(u \cos \psi+v \sin \psi)]\} F_{\phi}(q) .
\end{aligned}
$$

Since the measured variances and spectra given by Han are averaged over the two perpendicular axes, the $(u \cos \theta+v \sin \theta)^{2}$ term in the function $Q_{1}$ accounting for a direction $\theta$ of image motion measurements, was substituted here with its mean $q^{2} / 2$.

Transformation of spatial PSD $F(u, v)$ into temporal spectrum $g(f)$ is performed (Martin 1987; Conan et al. 1995) by integration of $F(u, v)$ over spatial frequency $v$ (across a wind), with a subsequent application of the Taylor transformation $u=f / V$ :

$g(f)=\frac{2}{V} \int_{-\infty}^{\infty} F(f / V, v) \mathrm{d} v, f \geq 0$.

Calculation of the integral (5) for small apertures $D \ll \rho h$ when $\operatorname{somb}^{2}(\pi D q) \approx 1$, and using equality (Gradstein \& Ryzhik 1971)

$\int_{0}^{\infty} \frac{\cos (a x)}{\left(\beta^{2}+x^{2}\right)^{-v+1 / 2}} d x=\frac{(2 \beta)^{v} \cos \pi v}{a^{v} \pi^{1 / 2}} \Gamma(\nu+1 / 2) K_{\nu}(a \beta)$

where $K_{v}$ is the modified Bessel function of the order $v$, yields

$$
\begin{aligned}
g(f)= & 2 c \operatorname{sinc}^{2}(\pi f T) f^{-p}\left[1-\frac{2(b f \sin \psi)^{p / 2}}{\Gamma(p / 2)}\right. \\
& \left.\times K_{p / 2}(2 b f \sin \psi) \cos (2 b f \cos \psi)\right] .
\end{aligned}
$$

Here

$b=\pi \rho h / V$,

$c=c_{\phi} \lambda^{2} \pi^{1 / 2} V^{p-1} \Gamma(p / 2) / \Gamma(1 / 2+p / 2)$.

In the high frequency (large separations $\rho$ ) domain $f \gg 1 / b$, the Bessel function is exponentially damping with $f$, so that (7) simplifies to

$g(f)=2 c \operatorname{sinc}^{2}(\pi f T) f^{-p}$.

Equation (9) evidently represents a doubled temporal PSD of the absolute image motion for integration time $T$. The structure of Eq. (9) allows one to identify the $c$-term defined by Eq. (8) with a factor normalizing the absolute image motion spectrum.

Comparison of Eqs. (7) and (9) shows that intrinsic features of the differential image motion spectrum are brought about by the factor marked in (7) by square brackets. To study its properties, we denote it (with the adjacent term $f^{-p}$ ) as a new function $\gamma(f)$. Rewriting Eq. (7) as

$g(f)=2 c \operatorname{sinc}^{2}(\pi f T) \gamma(f), D \ll \rho h$

we notice that $\gamma(f)$ is equal to the PSD $g(f)$ at $T=0$ with a numerical calibration $c=1 / 2$. The shape of the function $\gamma(f)$ depends on the positional angle $\psi$. In Fig. 3, $\gamma(f)$ is plotted for orientation of star pairs almost along the wind $\left(\psi=0.1^{\circ}, 10^{\circ}\right)$, across it $(\psi=\pi / 2)$, and when $\gamma(f)$ is averaged over $\psi$ in the range $0-2 \pi$. Each type of PSD shape in Fig. 3 corresponds to some peculiar condition that we specify below. Thus, occurance of the first three (non-averaged over $\psi$ ) PSDs is limited to 


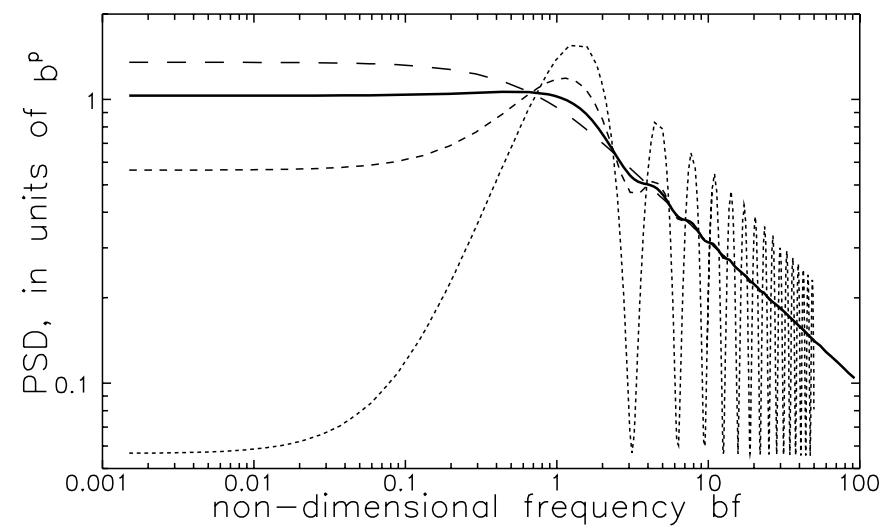

Fig. 3. The temporal PSD of differential image motion (7) at $c=1 / 2$ and $T=0$ (the function $\gamma(f)$ ), in units of $b^{p}$ versus a non-dimensional frequency $b f$. The function is plotted at $p=0.5, \psi=\pi / 2$ (long dash), $\psi=10^{\circ}$ (dash) and $\psi=0.1^{\circ}$ (short dash). The function $\hat{\gamma}(f)$ (averaged over $\psi$ ) is shown by a thick line.

the particular case of the single-layer turbulence, and observations of a single star pair. In other cases, the PSD $\hat{\gamma}(f)$ averaged over $\psi$ is detectable. The averaging over $\psi$ refers to observations of a few randomly (with respect to $\psi$ ) oriented star pairs, with a single- or multi-layer turbulence (which is a case studied by Han). In a multilayer turbulent atmosphere, detection of $\hat{\gamma}(f)$ - like PSDs is common, even for observations of a single arbitrary oriented star pair; an averaging effect is produced here by the changes of wind direction with height.

At high frequencies, the function $\gamma(f)$ oscillates around its mean $\hat{\gamma}(f)$ with an asymptote $g(f) \sim f^{-p}$. The amplitude of oscillations is higher at small $\psi$, and rapidly decreases with an increase in $\psi$. A deep modulation in PSDs, like that plotted for $\psi=0.1^{\circ}$, of course, cannot be registered in observations, because both the layered structure of the atmosphere and a certain dispersion of the wind direction produce the effect of averaging which suppresses small $\psi$ components. With the effective $\psi_{\text {eff }}$ about $10^{\circ}$, modulations become moderately weak (Fig. 3).

In the $f \ll 1 / b$ frequency domain, expansion of the function (7) into powers of $f$ yields

$$
\begin{aligned}
g(f)= & 2 c \operatorname{sinc}^{2}(\pi f T)\left\{(b \sin \psi)^{p} \frac{\Gamma(1-p / 2)}{\Gamma(1+p / 2)}\right. \\
& \times\left[1+2 b^{2} f^{2}\left(\frac{\sin ^{2} \psi}{2+p}-\cos ^{2} \psi\right)\right] \\
& \left.+2 b^{2} f^{2-p}\left(\cos ^{2} \psi-\frac{\sin ^{2} \psi}{2-p}\right)\right\}
\end{aligned}
$$

The amplitude of $\gamma(f)$ at $f=0$ is proportional to $\sin ^{p} \psi$. For stars oriented exactly along the wind, the PSD increases proportionally to $f^{2-p}$, which is in agreement with theoretical results by Martin (1987) and Conan et al. (1995) drawn at $p=2 / 3$. At orthogonal position of stars, the PSD is slowly decreasing with $f$, and up to $f \simeq 1.0 b^{-1}$ is approximately a white noise spectrum.

Averaging of Eq. (11) over $\psi$ results in

$$
\hat{\gamma}(f)=b^{p}\left[\gamma_{0}+\frac{1-p}{2-p}(b f)^{2-p}-\frac{2 \gamma_{0}}{(1+p)^{2}}(b f)^{2}\right]
$$

where $\gamma_{0}=\pi^{-1 / 2} \Gamma(1-p / 2) \Gamma(1 / 2+p / 2) \Gamma^{-2}(1+p / 2)$. At low frequencies, the $\hat{\gamma}(f)$ function (thick line in Fig. 3) approximately is a flat white noise spectrum with a small maximum at $(b f)^{p} \simeq 0.25(1-p)(2+p)^{2} / \gamma_{0}$, or $f \simeq(1-p) / b$. At $f=0$ there is a shallow spectral gap, which is better displayed in Fig. 5 on non-logarithmic axes where the upper curve is the same $\hat{\gamma}(f)$ function as that shown by a thick line in Fig. 3. The magnitude of the peak is only $0.036 \gamma_{0}$ at $p=1 / 3$, yet smaller, $0.024 \gamma_{0}$ at $p=2 / 3$, and vanishes at $p=1$.

Note that $g(f)$ not can be directly compared to the function $G(f)$ due to the different nature of the data they are formed upon. Really, while $g(f)$ refers to some continuous measured quantity and therefore is defined for any $f \geq 0, G(f)$ is calculated on the basis of the data sampled with a rate $\Delta t$. That limits the function $G(f)$ frequency domain by the bandwidth $0-f_{\mathrm{N}}$. Correct comparison of the two spectra requires taking into account the well-known aliasing effect (e.g. Vinnichenko et al. 1976), due to which the frequency component of $g(f)$ at $f>f_{\mathrm{N}}$ is imaged in a discrete spectrum as a low-frequency harmonic. Conversion of $g(f)$ into the observed PSD $\hat{G}(f)$ is found by summing

$$
\begin{aligned}
\hat{G}(f)= & g(f)+g\left(2 f_{\mathrm{N}}-f\right)+g\left(2 f_{\mathrm{N}}+f\right) \\
& +g\left(4 f_{\mathrm{N}}-f\right)+g\left(4 f_{\mathrm{N}}+f\right)+\ldots, f \leq f_{\mathrm{N}} .
\end{aligned}
$$

Performing the integration of Eqs. (7) or (13) over $f$ yields the variance of differential image motion

$\Delta^{2}=\int_{0}^{\infty} g(f) \mathrm{d} f=\int_{0}^{f_{\mathrm{N}}} \hat{G}(f) \mathrm{d} f$.

\section{Fitting of the measured temporal spectrum}

Now, proceeding to a quantitative consideration, we use Eqs. (7) and (13) in order to fit the observed spectrum $G(f)$ with the model function $\hat{G}(f)$. Only a single-layer turbulence was considered, which, nevertheless, yielded a good result. Expressions for $\hat{G}(f)$ depend on $\psi, \rho$ and three parameters $c$, $h / V$ and $p$ related to the turbulent layer. In view of a rather small $\rho$ spread in values, for simulation purposes we used its mean $\rho=10^{\prime}$. Also, due to a random orientation of star pairs, the function $\hat{G}(f)$ was averaged over a positional angle $\psi$ which thus dropped out of consideration. When finding parameters $c, h / V$ and $p$, we required best representation of the observed PSD shape (minimum squared deviation) in all frequency ranges. The integral $\int_{0}^{f_{\mathrm{N}}} \hat{G}(f) \mathrm{d} f$ was claimed to be equal to the observed variance of differential image motion, whose value, $0.0093 \mathrm{arcsec}^{2}$, was found both numerically by integration of the PSD shown in Fig. 2 of this paper, and directly from Fig. 2 given by Han (1989) as $\Delta$ taken at the exposure $T_{1}=1.2 \mathrm{~s}$.

Using a fixed $p=2 / 3$, the best fit is achieved with $c=$ $0.003105 \operatorname{arcsec}^{2} \mathrm{~Hz}^{-1 / 3}$ and $h / V=1700 \mathrm{~s}$. The relevant function $\hat{G}(f)$ (Fig. 2, dashed line) fits observations relatively well, but some deviations, such as a positive offset at high frequencies mounting to $40 \%$ of $G(f)$, may be seen.

Better results are achieved when $p$ is considered as a free parameter of simulation. It was found that to within random 
fluctuations of measurements a good, practically equal quality of fitting is obtained with any $p$ in the limits of $0.9-1.0$. Fluctuations in the PSD do not allow us to determine the $p$ value more accurately, therefore in future calculations we assume $p=0.94$. Thus, the best parameters fitting the measured $G(f)$ and representing the atmospheric turbulence are

$p=0.94, h / V=1130 \mathrm{~s}, c=0.001949\left(^{\prime \prime}\right)^{2} \mathrm{~Hz}^{-0.06}$.

Parameters $h$ and $V$ not can be determined separately. Assuming $V=15 \mathrm{~m} \mathrm{~s}^{-1}$, we estimate $h=17 \mathrm{~km}$ which places the turbulence in the stratosphere. Note that such a distinctive feature of the function $G(f)$ shape as a steep slope over a wide spectral region $0.05-0.25 \mathrm{~Hz}$ not can be simulated with $p=2 / 3$, even considering the atmosphere with $2-3$ turbulent layers. Of course, this does not mean a null turbulence at low altitudes because its contribution in PSD is attenuated due to the dependence $g(f) \sim h^{p}$ stated by Eq. (11).

It is interesting to note that in spite of a large difference in $p$ values (2/3 and 0.94), the PSDs plotted in Fig. 2 do not differ much in their form. This is a consequence of a relatively narrow range of the frequency $f$ variations as a result of which observed non-Kolmogorov PSDs may be sometimes misidentified as classic PSDs. To avoid confusion, a careful interpretation of the data is required.

\section{Dependence of the image motion variance on $\rho$}

In the previous section we used the observed temporal PSD in order to determine parameters of the turbulent layer. Now, using atmospheric parameters (15) and Eqs. (7) and (14), we calculate $\Delta$ as a function of the star separation $\rho$. The calculated function (curve 2, Fig. 1) allows us to verify how well the model acconts for a change of the parameter $\rho$ which until now was assumed to be a fixed quantity. One can see that both this curve and the line 1 originally plotted by Han fit observations almost equally well. This fact supports correctness of the present simulation in which the information given in Fig. 1 was not used at all. Besides, while parameters (15) were found from the data obtained with a short $1.2 \mathrm{~s}$ exposure, Fig. 1 refers to much longer $T=10 \mathrm{~s}$.

Note that curve 2 is not a straight line, as in the asymptotic case. Its slope depends on $\rho$, is about 0.43 at $\rho=10^{\prime}$, and decreases to the "conventional" $1 / 3$ value at $\rho=25^{\prime}$. Deviation from the asymptotic power law $\Delta \sim \rho^{p / 2}$ (line 3 with a slope of $p / 2=0.47$ ) is due to a violation of the asymptotic condition $\rho h / V \ll T$ at wide $\rho$, thus a linear approximation of the observed data in Fig. 1 seems to be invalid.

The individual scatter of points in Fig. 1 is caused partially by random errors which, according Han (1989), are small. Therefore we may suppose that the scatter is due primarily to different orientations $\psi$ of star pairs, and check this assumption by computations. Unfortunately, no data on $\psi$ are given by Han. Therefore the effect in $\Delta$ caused by the dispersion of $\psi$ we estimated only tentatively, with the assumption that $\psi$ angles changed in the range from their possible minimum value $\psi_{\mathrm{eff}}=10^{\circ}$ (Sect. 3) to $90^{\circ}$. The functions corresponding to these extreme angles (the curves 4 and 5) have been calculated with the integration of Eq. (7) over $\psi$. An offset of curves 4

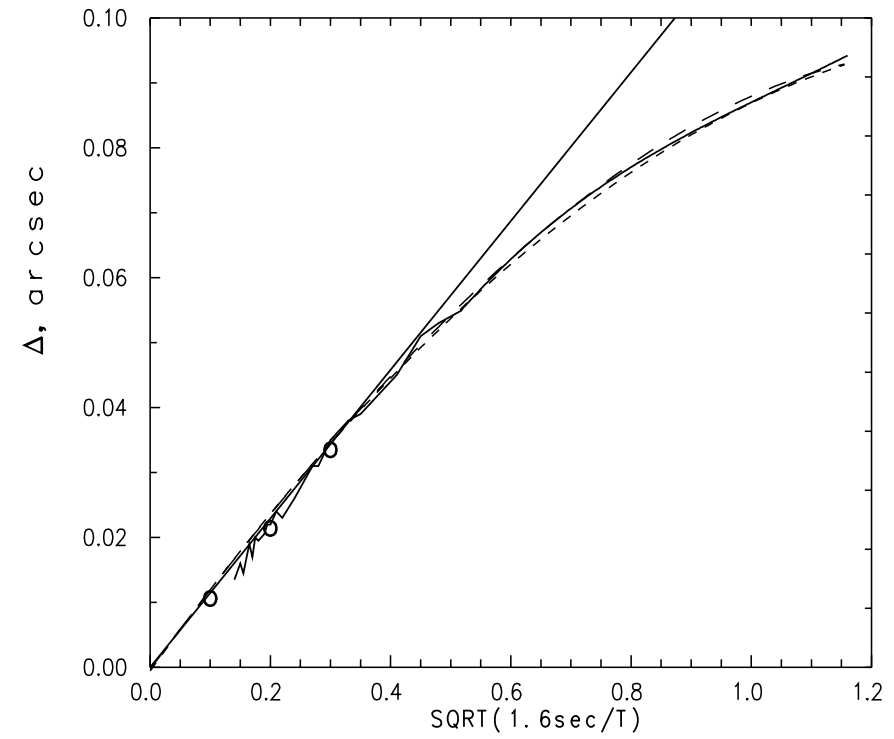

Fig. 4. The variance $\Delta$ versus integration time $T$ at $\rho=10^{\prime}$. Observed dependence (broken line); the simulated functions: with atmospheric parameters (14) - long dashed, with $p=2 / 3-$ short dashed, with the model (17) and outer scale $L_{0}=1800 \mathrm{~m}$ - open circles. Straight line is the asymptotic dependence at $\rho h / V \ll T$.

and 5 from the mean line 2 is large at small $\rho \approx 5-10^{\prime}$ (about $20-30 \%$ of $\Delta$ ), and almost vanishes at $\rho \approx 50^{\prime}$. We can see that most of experimental points are in the predicted limits; thus, the observed scatter of $\Delta$ values is really caused largely by different orientations of star pairs.

\section{Dependence of the image motion variance on $T$}

The use of parameters (15) allows us also to adequately predict the dependence of $\Delta$ on the exposure $T$. This is demonstrated by Fig. 4 , where our numerical results (long dashed line) found by integration (14) of the PSD (7) are compared to the observed data (broken curve). Performing calculations, we adopted the mean separation $\rho=10^{\prime}$ between stars. As noted above, the change of effective integration time $T$ was achieved by averaging measurements over some $m$ sequential periods of the MAP rulling. In the original study, however, a small difference between the $m \Delta t$ value and $T$ expressed by Eq. (1) was not taken into account. Therefore Han's experimental points (his Fig. 2) were transferred into Fig. 4 with corrections (1). The difference between $m \Delta t$ and $T$ is small and essential only at $m \leq 3$. Within statistical fluctuations, the calculated curve fits observations well enough. Here, as well as in Fig. 1, the measured behaviour is correctly predicted over a wide range of the argument variation, and under a strong nonlinearity of the function examined (the asymptotic dependence $\Delta \sim \sqrt{1 / T}$ is shown in Fig. 4 by a straight line). This is an extra confirmation of the validity of a model based on a different data source, a temporal spectrum of differential image motion.

It is interesting to note that equally good fitting is achieved with $p=2 / 3$ and corresponding parameters $c$ and $h / V$ (short dash). Both curves calculated with $p=2 / 3$ and $p=0.94$ are 
practically identical over a given range of the argument change and they diverge at $T$ much smaller than used in observations.

\section{A model of the temporal PSD allowing for an outer scale of turbulence}

\subsection{Theoretical considerations}

Figure 2 shows that the measured temporal spectrum at low $f \leq 0.1 \mathrm{~Hz}$ frequencies is approximately a flat white noise spectrum. One can notice however that slight positive fluctuations are present here at $f<0.01 \mathrm{~Hz}$, turning into a shallow spectral gap at $f \approx 0.01-0.02 \mathrm{~Hz}$. Most likely, both features are caused by random noise in the data. Nevertheless, we will try to consider now whether some details of this low-frequency microstructure of the observed spectrum can be explained by assuming a finite outer scale of turbulence $L_{0}$ which, as is known, may affect this frequency band. Introduction of $L_{0}$ in the model will allow us to determine its probable value, or at least establish a lower limit of $L_{0}$. Note that frequencies $0.01-0.02 \mathrm{~Hz}$ at $V=15 \mathrm{~m} \mathrm{~s}^{-1}$ correspond to scales of $750-1500 \mathrm{~m}$, which are comparable to some known estimates of $L_{0}$ covering a range from $5 \mathrm{~m}$ (Coulman et al. 1988) to 100-1000m (Vinnichenko et al. 1976).

Saturation of turbulence at large scales is described by a von Karman model of 3-D refractivity spectrum $\Phi_{n}(q) \sim$ $\sqrt{q^{2}+L_{0}^{-2}}-11 / 3$ (e.g. Goodman 1985). This spectral model can be considered formally as a modification of the corresponding Kolmogorov expression $\Phi_{n}(q) \sim q^{-11 / 3}$, which is performed by substitution of $q$ on a new spatial frequency

$\hat{q}=\sqrt{q^{2}+L_{0}^{-2}}$

restricted below with $L_{0}^{-1}$. A 2D power spectrum of a phase $F_{\phi}(q)$ in the von Karman model therefore differs from the Kolmogorov one $F_{\phi}(q) \sim q^{-11 / 3}$ also with use of the frequency $\hat{q}$. In further calculations thus we adopted a model

$F_{\phi}(q)=c_{\phi} \sqrt{q^{2}+L_{0}^{-2}}-3-p$

which corresponds to the von Karman spectrum except for use of a variable power, and inherits a structure of Eq. (3). At $L_{0} \rightarrow \infty$, Eqs. (3) and (17) are equivalent.

Convertion to temporal frequencies is performed as described in Sect.3 and involves integration (5) of expression (4), where $F_{\phi}(q)$ is given by (17), with respect to the frequency $v$. To obtain the result, let us represent a term $q^{2} F_{\phi}(q)$ in Eq. (4) as

$q^{2} F_{\phi}(q) \sim \frac{q^{2}}{\hat{q}^{3+p}}=\frac{1}{\sqrt{\hat{u}^{2}+v^{2}} 1+p}-\frac{L_{0}^{-2}}{\sqrt{\hat{u}^{2}+v^{2}} 3+p}$

where $\hat{u}^{2}=u^{2}+L_{0}^{-2}$. Comparison of the first term in Eq. (18) with an expression $q^{2} F_{\phi}(q) \sim 1 / \sqrt{u^{2}+v^{2}} 1+p$ valid for the model (3) shows that the only difference is in the use of frequencies $\hat{u}$ and $u$. Therefore we may utilize the results of integration over $v$ previously derived in Sect. 3, replacing only $u$ by $\hat{u}$. The following Taylor transformation $u=f / V$ then leads to the expression having the structure of Eq. (7) where $f$ is substituted (except terms $\cos (2 b f \cos \psi)$ and $\left.\operatorname{sinc}^{2}(\pi f T)\right)$ for a modified frequency

$\hat{f}=\sqrt{f^{2}+f_{0}^{2}}$

where $f_{0}=V / L_{0}$ is a measure of $L_{0}$ in a temporal scale. Processing of a second term in Eq. (18) is accomplished in the same way but by replacing $p$ by $p+2$.

Finally, we derive a temporal PSD of differential image motion with allowance for $L_{0}$ :

$$
\begin{aligned}
g(f)= & 2 c \operatorname{sinc}^{2}(\pi f T) \hat{f}^{-p}\left[1-\frac{p f_{0}^{2}}{(p+1) \hat{f}^{2}}\right. \\
& -\frac{2(b \hat{f} \sin \psi)^{p / 2}}{\Gamma(p / 2)} K_{p / 2}(2 b \hat{f} \sin \psi) \cos (2 b f \cos \psi) \\
& +\frac{4(b \hat{f} \sin \psi)^{p / 2+1}}{(p+1) \Gamma(p / 2)} \frac{p f_{0}^{2}}{\hat{f}^{2}} K_{p / 2+1}(2 b \hat{f} \sin \psi) \\
& \times \cos (2 b f \cos \psi)] .
\end{aligned}
$$

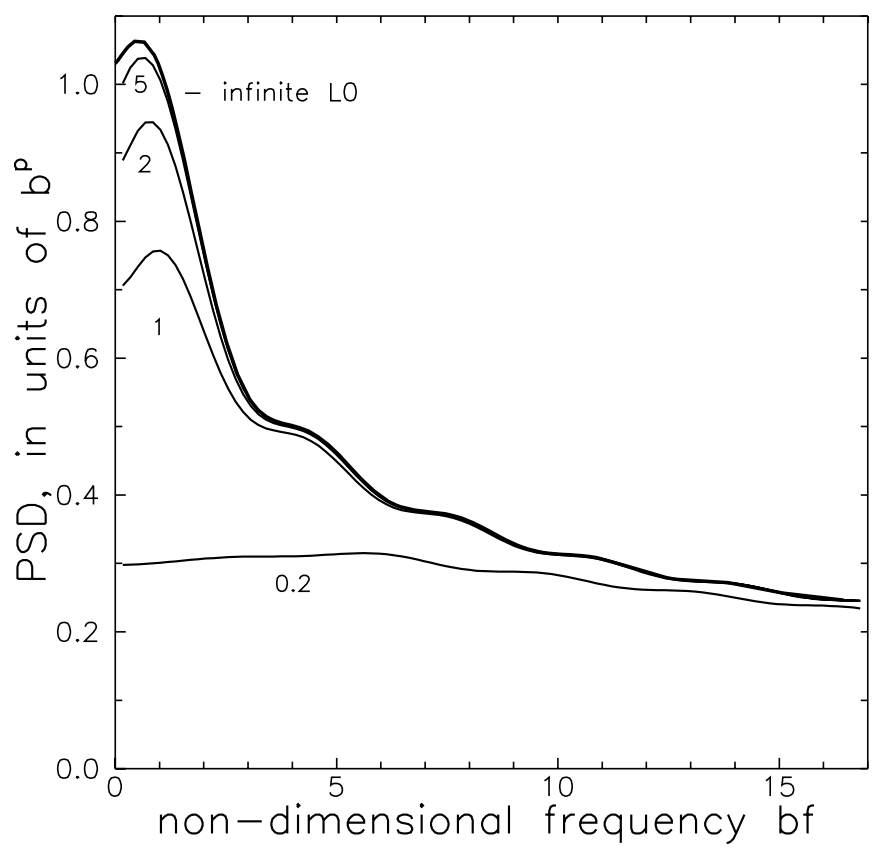

Fig. 5. The temporal spectrum of differential image motion based on the model (17) at $p=0.5, c=1 / 2$ and $T=0$ versus a nondimensional frequency $b f$. The functions are plotted in descending order for outer scales $L_{0}=\infty$ (upper thick line), $5 b V, 2 b V, b V$ and $0.2 b V$ (lower curve); numerical averaging with respect to the winddependent angle $\psi$ was applied.

Figure 5 shows the function (20) behavior for several outer scale lengths from $5 b V$ to $0.2 b V$ (corresponding to $f_{0}$ from $0.2 b^{-1}$ to $\left.5 b^{-1}\right)$. As in Fig. 3, calculations were executed with parameters $p=0.5, c=1 / 2, T=0$, and numerically averaged with respect to the position angle $\psi$. The upper curve corresponds to the case of infinite scale analyzed in Sect. 3 and is 
shown in Fig. 3 with a thick line. The lower curve plotted at very short $L_{0}<b V$ is of a form typical of the (doubled) absolute image motion PSD, which is valid at large separations $\rho h \gg V T$

$g(f)=2 c \operatorname{sinc}^{2}(\pi f T){\sqrt{f^{2}+f_{0}^{2}}}^{-p}\left[1-\frac{p}{p+1} \frac{f_{0}^{2}}{f^{2}+f_{0}^{2}}\right]$.

A shape of the function $g(f)$ varies depending upon a relative magnitude of $L_{0}$ and a linear size $b V=\pi \rho h$ of a line connecting the two stars in projection on the turbulent layer. For long $L_{0} \gg b V$, the effect is weak (Fig. 5) resulting mainly in a decrease of spectral height at low frequencies $f \leq b^{-1}$; at $f \gg b^{-1}$ there is practically no noticable effect.

In the case of $L_{0}<b V$ or $f_{0}>b^{-1}$ the effect is evidently strong (lower curve), but also in the low frequency range. Here, at $f \leq 2 f_{0}$, the PSD is reduced to a height of about $2 c \operatorname{sinc}^{2}(\pi f T)\left(2 f_{0}\right)^{-p}$; at $f>2 f_{0}$ the asymptotic law (9) remains valid.

Note that the shape of the function $g(f)$ in cases of either long or short $L_{0}$ is similar in that they both have flat white noise and (9) asymptotes; the difference is that a knee frequency separating these loci occurs at physically different frequencies $b^{-1}=V /(\pi \rho h)$ and $f_{0}=V / L_{0}$ (dependent or not dependent on angular separation between the stars $\rho$ ).

\subsection{Fitting of the MAP data}

The model (17) discussed in the above subsection was applied to the fitting of the observed power spectrum $G(f)$ so as to extract probable values of $L_{0}$ and $f_{0}$ parameters. A solution was found at $f_{0}=0.08 b^{-1} \approx 0.008 \mathrm{~Hz}$ and parameters (15). The model PSD matches the PSD obtained previously (thick line in Fig. 2) except at low frequencies where a new function is shown by open circles. The quality of fitting rapidly deteriorates with the increase of $f_{0}$, therefore a value $0.008 \mathrm{~Hz}$ can be considered rather as the upper limit of $f_{0}$. As seen from Fig. 2, both models (3) corresponding to $f_{0}=0$ and (17) yield equally good fitting, and no one can be favoured due to noise in the experimental data. Thus, for this particular stratospheric layer detected at $h \approx 17 \mathrm{~km}$, we fix limits

$$
\begin{aligned}
& 0 \leq f_{0} \leq 0.008 \mathrm{~Hz} \\
& \infty \geq L_{0} \geq 1800 \mathrm{~m}
\end{aligned}
$$

where tranformation to spatial scales $L_{0}=V / f_{0}$ was performed assuming $V=15 \mathrm{~m} \mathrm{~s}^{-1}$.

In Fig. 4, by open circles we show a bias in the dependence of the variance $\Delta$ on integration time $T$ caused by the presence of outer scale (22); a new corrected dependence is in a slightly better agreement with experimental data.

\section{Conclusions}

The sample estimate $p=0.9-1.0$ derived in this study and corresponding to the particular stratospheric layer is larger than the typical $1 / 3-2 / 3$ values found from observations with infrared interferometers (Bester et al. 1992; Busher et al. 1995). Large $p$ values were detected, for example, by Belen'kii et al. (1997) who observed Polaris image motion with a telescope bolted in place so as to minimize uncontrolled telescope movements. For the tilt power spectra they found approximately a -1 slope in the low frequency range, which corresponds to $p=1$.

The rather close estimates $p=0.97$ and $0.01<f_{0}<$ $0.025 \mathrm{~Hz}\left(1500<L_{0}<600 \mathrm{~m}\right.$ at $\left.V=15 \mathrm{~m} \mathrm{~s}^{-1}\right)$ had been derived earlier by the author (Lazorenko 1992), who analyzed results of photographic and visual studies of the absolute image motion obtained for site testing programs in the former USSR. It should be noted, however, that absolute image motion data refer rather to the low altitude turbulence which is usually stronger and is not attenuated by the factor $h^{p}$ present in expression (11) for differential measurements.

Our aim was to derive analytical expressions for a temporal image motion spectrum, and apply them to the MAP experimental data. A restoration of atmospheric turbulent parameters from the differential image motion spectrum appears to be succesful due to the presence in the measured spectrum of both the white noise locus and the exponentially decreasing region (corresponding to the absolute image motion). The spectral data had not been used in the original study (Han 1989), the results obtained were based on the fit of the $\Delta(\rho)$ function alone. This limitation, combined with a significant scatter of measured points in Fig. 1 (probably caused by a dispersion of $\psi$ angles), non-linearity of the function $\Delta(\rho)$, and a narrow range of $\rho$ change, resulted in a biased estimate of $p$.

Concerning the measurements in wide fields $D \ll \rho h$, we notice that the shape of functions $\Delta(\rho), \Delta(T)$ and $G(f)$ is not critically sensitive to the actual value of $p$. This is demonstrated by Figs. 1, 2 and 4 where the observed data was well fitted with different $p$. We conclude that:

1) Sufficiently good predictions of $\Delta$ to be used in practical applications can be derived in a conventional Kolmogorov model, with no loss of accuracy even at large deviations of $p$ from $2 / 3$. Thus, the empirical dependence $\Delta(\rho)$ derived in Paper I is still valid for moderate $\rho$;

2) In image motion studies, the non-classic distortions of phase are detected with difficulty and are easily confused with the classic distortions.

Acknowledgements. The author thanks the anonymous referee for useful comments concerning outer scale effects in the image motion data.

\section{References}

Belen'kii, M. S., Karis, S. J., Brown, J. M., \& Fugate, R. Q. 1997, SPIE Proc., 3126, 113

Bester, M., Danchi, W. C., Degiacomi, C. G., et al. 1992, ApJ, 392, 357

Buscher, D. F., Armstrong, J. T., Hummel, C. A., et al. 1995, Appl. Opt., 34, 1081

Conan, J., Rousset, G., \& Madec, P. 1995, JOSA A, 12, 1559

Fried, D. L. 1965, JOSA, 55, 1427

Gatewood, G. D. 1987, AJ, 94, 213

Goodman, J. W. 1985, Statistical optics (Wiley-Interscience publication, New York) 
Gradstein, I. S., \& Ryzhik, I. M. 1971, The tables of integrals, sums, series and products (Nauka, Moscow)

Han, I. 1989, AJ, 97, 607

Hog, E. 1968, Z. Astrophys., 69, 313

Hogstrom, U., Smedman, A., \& Bergstrom, H. 1998, J. Atmos. Sci., 56,959

Lazorenko, P. F. 1992, Kin. Phys. Celest. Bodies, 3, 78

Lazorenko, P. F. 2002, A\&A, 382, 1125
Lindegren, L. 1980, A\&A, 89, 41

Martin, H. M. 1987, PASP, 99, 1360

Sarazin, M., \& Roddier, F. 1990, A\&A, 227, 294

Tatarsky, V. I. 1961, Wave propagation in a turbulent medium (Dover, New York)

Vinnichenko, N. K., Pinus, N. Z., Shmeter, S. M., \& Shur, G. N. 1976, The turbulence in a free atmosphere (Hydrometeoizdat, Leningrad) 